\section{Effects of Au}

Nanoparticles on $\mathrm{TiO}_{2}$ in the Photocatalytic Degradation of an Azo Dye

\author{
Marta Mrowetz ${ }^{1}$, Alberto Villa², \\ Laura Prati², Elena Selli ${ }^{*}$ \\ ${ }^{1}$ Dipartimento di Chimica Fisica ed Elettrochimica and \\ CIMAINA, Università degli Studi di Milano, Via Golgi \\ 19, I-20133 Milano, Italy \\ 2 Dipartimento di Chimica Inorganica Metallorganica \\ e Analitica and Centro CNR, Università degli Studi di \\ Milano; Via Venezian 21, I-20133 Milano, Italy \\ * To whom correspondence should be addressed \\ E-mail: elena.selli@unimi.it
}

\begin{abstract}
The photocatalytic activity of Au modified titanium dioxide was evaluated in the photodegradation of the azo dye Acid Red 1 (AR1) under $254 \mathrm{~nm}$ irradiation. Noble metal nanoparticles were deposited on $\mathrm{TiO}_{2}$ either through depositionprecipitation (DP), or by immobilisation of preformed metallic sols (polyvinylalcohol (PVA)/ $\mathrm{NaBH}_{4}$ or tetrakis(hydroxymethyl)phosphonium chloride (THPC)/NaOH systems). Gold nanoparticles on the photocatalyst surface had dimensions of around 3-4 $\mathrm{nm}$ in diameter, as determined by HRTEM analysis, and exhibited visible light plasmon absorption. THPC Au/TiO appears to be the most photoactive amongst the photocatalysts with a $1 \mathrm{wt} . \% \mathrm{Au}$ loading, while among THPC samples with different Au loadings (0.5-20.0 wt.\%) the maximum photoactivity was attained with $5 \mathrm{wt} . \% \mathrm{Au} / \mathrm{TiO}_{2}$. The higher AR1 photodegradation rate observed on $\mathrm{Au} / \mathrm{TiO}_{2}$ at basic $\mathrm{pH}$ can be related to the higher concentration of hydroxyl anions at the interface: these are able to effectively scavenge photoproduced valence band holes, possibly in competition with $\mathrm{Au}^{0}$ oxidation to $\mathrm{Au}^{+}$.
\end{abstract}

\section{Introduction}

Photocatalysis on semiconductor oxides has been widely investigated in recent years, mainly because of its high potential for ensuring the complete destruction of organic contaminants both in the aqueous and in the gas phase (1). Titanium dioxide is by far the most extensively studied photocatalytic material, thanks to its outstanding physical properties, high resistance to dark and photoinduced corrosion, easy availability and low cost. Upon photoinduced band gap excitation, electrons are promoted in the semiconductor conduction band and holes are consequently generated in its valence band; such charge carriers are able to reduce and oxidise many species adsorbed on the semiconductor particles and to induce the oxidative destruction of organics up to their overall mineralization, i.e. conversion into $\mathrm{CO}_{2}, \mathrm{H}_{2} \mathrm{O}$ and mineral acids. The high rate of recombination between photogenerated electron/ hole pairs is a major rate-determining factor controlling photocatalytic efficiency (2).

Noble metal deposition can improve the photocatalytic efficiency of titanium dioxide, by increasing electron/hole separation. For example, small platinum islands deposited on the semiconductor surface have been shown to efficiently compete with the undesired electron-hole recombination reaction, favouring hole trapping and successive oxidation reactions (3). This effect was attributed to electron capture by $\mathrm{Pt}^{0}$, rather than to a catalysed oxygen reduction by conduction band electrons (4), i.e. the noble metal deposit can act as a sink for photopromoted electrons (5). In fact, because of the high electronegativity of metal nanoparticles, the Fermi level can be shifted to negative potentials, with a consequent increased accumulation of electrons. Thus, the equilibration of the Fermi-level between the metal itself and the semiconductor favours electron accumulation in the composite metal/ $/ \mathrm{TiO}_{2}$ system (6).

The deposition of gold nanoparticles on the titanium dioxide surface was explored in the present study as a means of improving its photocatalytic activity. Three different deposition methods were employed, in order to optimize the deposition procedure. In addition to the well-known deposition-precipitation (DP) technique, first used by Haruta (7), the use of metal colloidal solutions was also examined as a way of controlling the size of gold nanoparticles (8), which can affect both the catalytic and photocatalytic performance of $\mathrm{Au} / \mathrm{TiO}_{2}$ systems. The various samples of $\mathrm{Au} / \mathrm{TiO}_{2}$ photocatalysts were evaluated in the photocatalytic degradation of the azo dye Acid Red 1 in the aqueous phase under UV irradiation. The effect of the Au loading on the $\mathrm{TiO}_{2}$ was also investigated, to maximise the beneficial role of gold on the processes occurring at the water-metalsemiconductor interface. Finally, the effect of $\mathrm{pH}$ on the photocatalytic activity of $\mathrm{Au} / \mathrm{TiO}_{2}$ was investigated to ascertain if and how the reactivity of trapped holes at the water-metalsemiconductor interface might be modified by alteration of the photocatalyst surface speciation. 


\section{Experimental section}

\section{Deposition of gold nanoparticles and $\mathrm{Au} / \mathrm{TiO}_{2}$ characterisation}

Gold foils of $99.9999 \%$ purity, purchased from Fluka, poly-vinylalcohol (PVA, 98\% hydrolysed, average $M_{r} 13-23 x$ $10^{3}$ ), tetrakis(hydroxymethyl)phosphonium chloride (THPC), purchased either from Fluka or from Aldrich, and $\mathrm{TiO}_{2}$ from Degussa (P25; 25\% rutile, 75\% anatase, surface area $50 \mathrm{~m}^{2}$ $\mathrm{g}^{-1}$ ) were used for the preparation of $\mathrm{Au} / \mathrm{TiO}_{2}$ samples. $\mathrm{NaOH}$ was $99.9 \%$ pure from Merck and was stored under nitrogen.

PVA - stabilised gold sols were obtained from an aqueous solution containing $100 \mathrm{mg} \mathrm{l}^{-1}$ of $\mathrm{HAuCl}_{4}$, prepared by dissolving $30 \mathrm{mg}$ of gold in a minimum amount of a 3:1 (vol/vol) $\mathrm{HCl} / \mathrm{HNO}_{3}$ mixture. After removing $\mathrm{HNO}_{3}$, the auric solution was diluted with distilled water and maintained under vigorous stirring while adding a 2 wt.\% PVA solution, up to PVA/Au (wt/wt) = 0.64. A 0.1 M freshly prepared $\mathrm{NaBH}_{4}$ solution was then added up to $\mathrm{NaBH}_{4} / \mathrm{Au}(\mathrm{mol} / \mathrm{mol})=5$, to form a ruby red metallic sol (9).

Sols generated in the presence of the THPC/NaOH system were prepared as reported elsewhere (10). A freshly prepared $0.05 \mathrm{M}$ THPC aqueous solution was added to a $10^{-3} \mathrm{M} \mathrm{NaOH}$ solution (THPC/Au (wt/wt) =0.95). After a few minutes a $10^{-3} \mathrm{M}$ $\mathrm{HAuCl}_{4}$ solution was added dropwise, forming a brown metallic sol. A reference sample was prepared following exactly the same procedure, apart from $\mathrm{HAuCl}_{4}$ addition.

The stabilised Au nanoparticles were immobilised on $\mathrm{TiO}_{2}$ by simply dipping the oxide support in the various metal dispersions, which were acidified up to $\mathrm{pH} 1.5$ - 2 by addition of $6 \mathrm{M} \mathrm{H}_{2} \mathrm{SO}_{4}$. The amount of $\mathrm{TiO}_{2}$ powder was calculated so as to have a final gold loading of $1.0 \mathrm{wt} . \%\left(\mathrm{PVA} \mathrm{Au} / \mathrm{TiO}{ }_{2}\right)$, or equal to $0.5,0.7,1.0,1.5,5.0,7.0,10$ and 20 wt.\% (THPC Au/ $\mathrm{TiO}_{2}$ ). After $1 \mathrm{~h}$ the slurry was filtered and the total absorption of gold was checked by ICP analysis of the filtrate. Before use, the photocatalysts were thoroughly washed with distilled water and then dried at $100^{\circ} \mathrm{C}$ for $2 \mathrm{~h}$.

Alternatively, $\mathrm{Au} / \mathrm{TiO}_{2}$ photocatalyst samples were prepared by the deposition-precipitation (DP) method (7). The $\mathrm{TiO}_{2}$ support was dispersed in water (approximately $10 \mathrm{ml} / \mathrm{g}$ of support) and a $0.3 \mathrm{M} \mathrm{NaOH}$ solution was added to raise the $\mathrm{pH}$ to 10 . The required amount of gold (in the form of $\mathrm{HAuCl}_{4}$ solution) was added dropwise under vigorous stirring over a time interval of $2 \mathrm{~h}$. The mixture was stirred for 30 min, centrifuged and washed with at least ten times its own volume of distilled water. The reduction of the catalyst was carried out by calcination at $400^{\circ} \mathrm{C}$ in air for $4 \mathrm{~h}$.

The effect of Au deposition on the crystalline structure of $\mathrm{TiO}_{2}$ Degussa P25 was analysed by X-ray diffraction employing a Philips PW 1820 powder diffractometer, operating at 40 $\mathrm{kV}$ and $40 \mathrm{~mA}$, with Ni-filtered Cu K $\alpha$ radiation ( $\lambda=1.5148$ $\AA)$. Electron micrographs of the samples were obtained by a Jeol 2000EX microscope, as already reported (11). XPS measurements were performed in an M-Probe Instrument (SSI) equipped with a monochromatic AI-K $\alpha$ source (1486.6 eV) with a $200 \times 750 \mu \mathrm{m}$ spot size and a $25 \mathrm{eV}$ pass energy.
The $\mathrm{Au} 4 \mathrm{f}$ region around $84 \mathrm{eV}$ was investigated in detail. The accuracy of the reported binding energies can be estimated to be $\pm 0.2 \mathrm{eV}$. A Lambda 19 Perkin-Elmer apparatus, equipped with a RSA-PE-19 accessory for reflectance measurements, was employed to collect DR UV-vis spectra.

\section{Photocatalysis tests}

Acid Red 1 (AR1, see Scheme 1) purchased from Aldrich, was purified by repeated crystallisation from methanol and its purity from organic contaminants verified by NMR analysis. All other chemicals used in the photocatalytic experiments were high purity Aldrich products and were employed as received. Water purified by Milli-Q water system (Millipore) was used throughout.

The degradation runs were carried out in the $800 \mathrm{ml}$ reactor previously described (12), equipped with an immersion Jelosil, model NSL15, $15 \mathrm{~W}$ low pressure mercury arc lamp, emitting exclusively at $253.7 \mathrm{~nm}$, with a $6.3 \times 10^{-6}$ Einstein $\mathrm{I}^{-1}$ $\mathrm{s}^{-1}$ radiation flow, as determined by ferrioxalate actinometry (13). The reactor was magnetically stirred during the runs and thermostated at $(30 \pm 1)^{\circ} \mathrm{C}$ by continuous water recirculation through an external glass jacket.

The irradiated aqueous suspensions contained $0.1 \mathrm{~g} \mathrm{l}^{-1}$ of $\mathrm{TiO}_{2}$ or $\mathrm{Au} / \mathrm{TiO}_{2}$ and an initial AR1 concentration around $2.5 \times 10^{-5} \mathrm{M}$. Prior to AR1 addition, the aqueous suspensions containing the photocatalysts were always sonicated for 30 min, employing an ultrasound source emitting at $20 \mathrm{kHz}$, produced by Stimin (Italy).

The $\mathrm{pH}$ of the suspensions was monitored during the photocatalytic runs by means of an Amel Instruments 334-B pH-meter. A pH decrease was observed under so-called natural $\mathrm{pH}$ conditions, i.e. when the initial $\mathrm{pH}$ of the AR1 aqueous suspensions was not adjusted, from an initial value of 5.8 to a final value of ca. 4.4 , as a consequence of the production of stable acids (14). The effect of $\mathrm{pH}$ on the rate of AR1 photocatalytic degradation rates was studied by adjusting the initial $\mathrm{pH}$ by addition of small amounts of concentrated $\mathrm{NaOH}$ and $\mathrm{HClO}_{4}$ solutions, which are known to have negligible influence on the photocatalytic activity (15).

2-ml samples were periodically withdrawn from the reactors and analysed, after removal of $\mathrm{TiO}_{2}$ particles by centrifugation at $3000 \mathrm{rpm}$ for $30 \mathrm{~min}$, employing an ALC

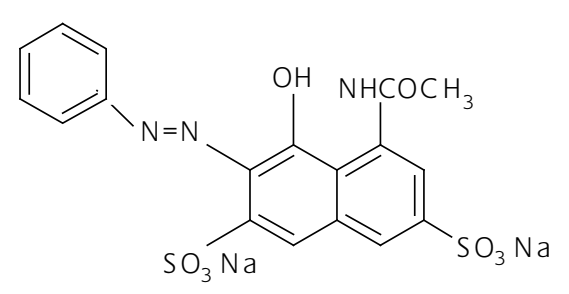

Scheme 1

Molecular structure of the azo dye Acid Red 1 (AR1) 


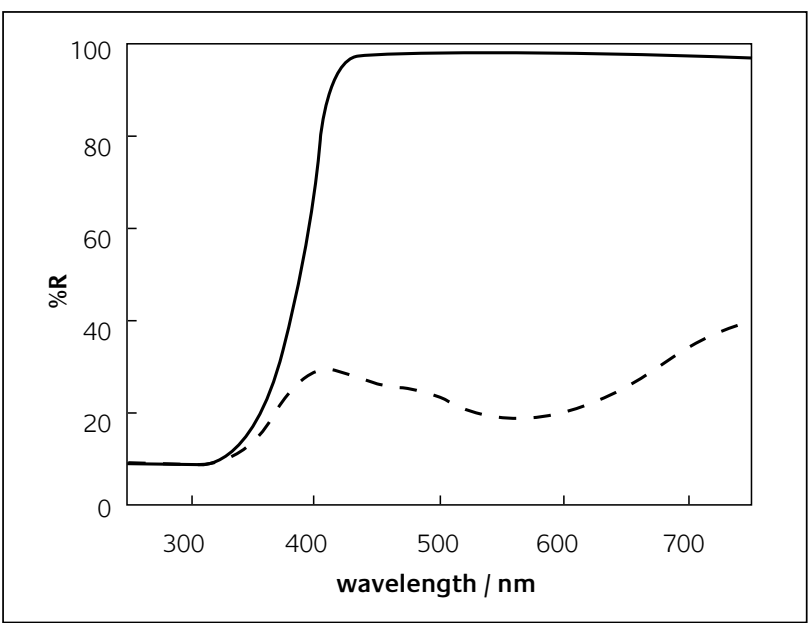

Figure 1

Diffuse reflectance spectra of unmodified $\mathrm{TiO}_{2}$ (solid line) and $5 \mathrm{wt} . \%$ $\mathrm{Au} / \mathrm{TiO}_{2}$ THPC (dashed line)

4225 centrifuge (16). The cleavage of the azo bond of AR1, leading to its bleaching (also mentioned as AR1 degradation), was monitored by spectrophotometric analysis at $531 \mathrm{~nm}$ (maximum AR1 absorption, $\varepsilon=(3.13 \pm 0.02) \times 10^{4} \mathrm{M}^{-1} \mathrm{~cm}^{-1}$ ) by means of a Perkin Elmer Lambda 16 spectrophotometer (16). The mineralisation of the substrate was detected using a total organic carbon (TOC) analyser (Shimadzu Instruments, TOC-5000A). All runs were repeated at least twice, to check their reproducibility.

AR1 adsorption was determined in aqueous suspensions containing $1.0 \mathrm{~g} \mathrm{l}^{-1}$ of $\mathrm{TiO}_{2}$ or Au modified $\mathrm{TiO}_{2}$ and a $2.5 \times 10^{-5}$ $\mathrm{M}$ initial AR1 concentration. After continuous stirring for $24 \mathrm{~h}$ in the dark at $30^{\circ} \mathrm{C}$, the photocatalyst was removed by centrifugation and the aqueous phase was analysed spectrophotometrically for AR1 residual content.

\section{Results and discussion}

\section{Photocatalysts characterisation}

Deposition-precipitation (DP) and two quite novel methods, employed for the first time to prepare $\mathrm{Au} / \mathrm{TiO}_{2}$ photocatalysts (17-21) and based on the reduction of $\mathrm{HAuCl}_{4}$, either with $\mathrm{NaBH}_{4}$ in the presence of polyvinylalcohol (PVA) (11) or with the tetrakis(hydroxymethyl)phosponium chloride (THPC)/NaOH system (8), were employed to deposit gold nanoparticles on the surface of titanium dioxide.

The deposition of gold nanoparticles on $\mathrm{TiO}_{2}$ was confirmed by the colour change of the modified oxide powder, turning from white into deep purple, a colour deriving from the surface plasmon resonance of $\mathrm{Au}^{0}$ islands (22). In fact, the diffuse reflectance UV-vis spectra of unmodified $\mathrm{TiO}_{2}$ and of $5 \mathrm{wt} . \%$ $\mathrm{Au} / \mathrm{TiO}_{2}$ THPC shown in Figure 1 indicate that, while unmodified titanium dioxide reflects strongly at wavelengths above 400 nm, Au-modified $\mathrm{TiO}_{2}$ exhibits a less evident absorption edge at $400 \mathrm{~nm}$, followed by the plasmon resonance absorption of Au nanoparticles at longer wavelengths. Light absorption by the deposited metal causes a collective oscillation of the
Table 1: Comparison between 1 wt.\% Au/TiO prepared by the DP, PVA and THPC method: diameter of gold nanoparticles, measured by HRTEM analysis, and first order rate constants of AR1 photocatalytic degradation

\begin{tabular}{lcc}
\hline Sample & $\mathbf{d}_{\mathrm{Au}}(\mathbf{H R T E M}) / \mathbf{n m}$ & $\mathbf{1 0}^{\mathbf{4}} \mathbf{x} \mathbf{~} / \mathbf{~ s}^{\mathbf{1}}$ \\
\hline THPC & 3.6 & $7.0 \pm 0.7$ \\
PVA & 3.5 & $6.4 \pm 0.3$ \\
DP & 4.2 & $6.0 \pm 0.2$ \\
\hline
\end{tabular}

free conduction band electrons of the gold nanoparticles as a consequence of their optical excitation (23), a phenomenon observed when the wavelength of the incident light far exceeds the particle diameter (24). The plasmon resonance, responsible for absorption in the visible region, does not induce any photocatalytic activity of $\mathrm{Au} / \mathrm{TiO}_{2}$ under visible light irradiation (21), because no holes are photoproduced in the semiconductor valence band under absorption by the metal nanoparticles deposited on the $\mathrm{TiO}_{2}$ surface.

Figure 2 shows the XRD patterns of unmodified $\mathrm{TiO}_{2}$ and of $1.0,5.0$ and 20 wt.\% Au/TiO 2 THPC samples. Gold deposition did not affect the anatase and rutile phases, which can be easily distinguished by the peaks at ca. $2 \theta=25.4^{\circ}$ and $27.5^{\circ}$, respectively. The THPC as well as the PVA methods implied gold deposition on $\mathrm{TiO}_{2}$ at room temperature and this guarantees the persistence of the optimal anatase to rutile ratio of Degussa P25, without any structural modification induced by thermal treatment. Au deposition was responsible for new peaks typical of crystalline gold at ca. $2 \theta=38.5^{\circ}$ and $44^{\circ}$ (25). Their intensity increased with Au loading, the Au phase being hardly detectable in 1 wt.\% Au, as previously reported (26).

High resolution XPS spectra of the $A u(4 f)$ region of the 1 wt.\% Au/TiO 2 THPC sample, recorded before and after 3 $\mathrm{h}$ irradiation at $254 \mathrm{~nm}$, are given in Figure 3. They exhibit two peaks at binding energies (BE) of ca. $83.6 \mathrm{eV}$ and 87.3 $\mathrm{eV}$, originating from $\mathrm{Au} 4 \mathrm{f}_{7 / 2}$ and $\mathrm{Au} 4 \mathrm{f}_{5 / 2}$ electrons of $\mathrm{Au}(0)$,

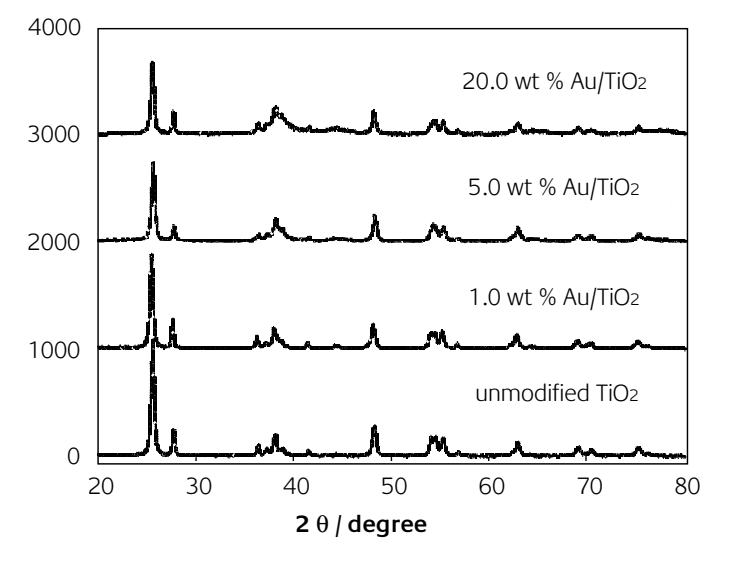

Figure 2

XRD patterns of unmodified TiO and of 1 wt.\%, 5 wt.\% and 20 wt.\% $\mathrm{Au} / \mathrm{TiO}_{2} \mathrm{THPC}$ 


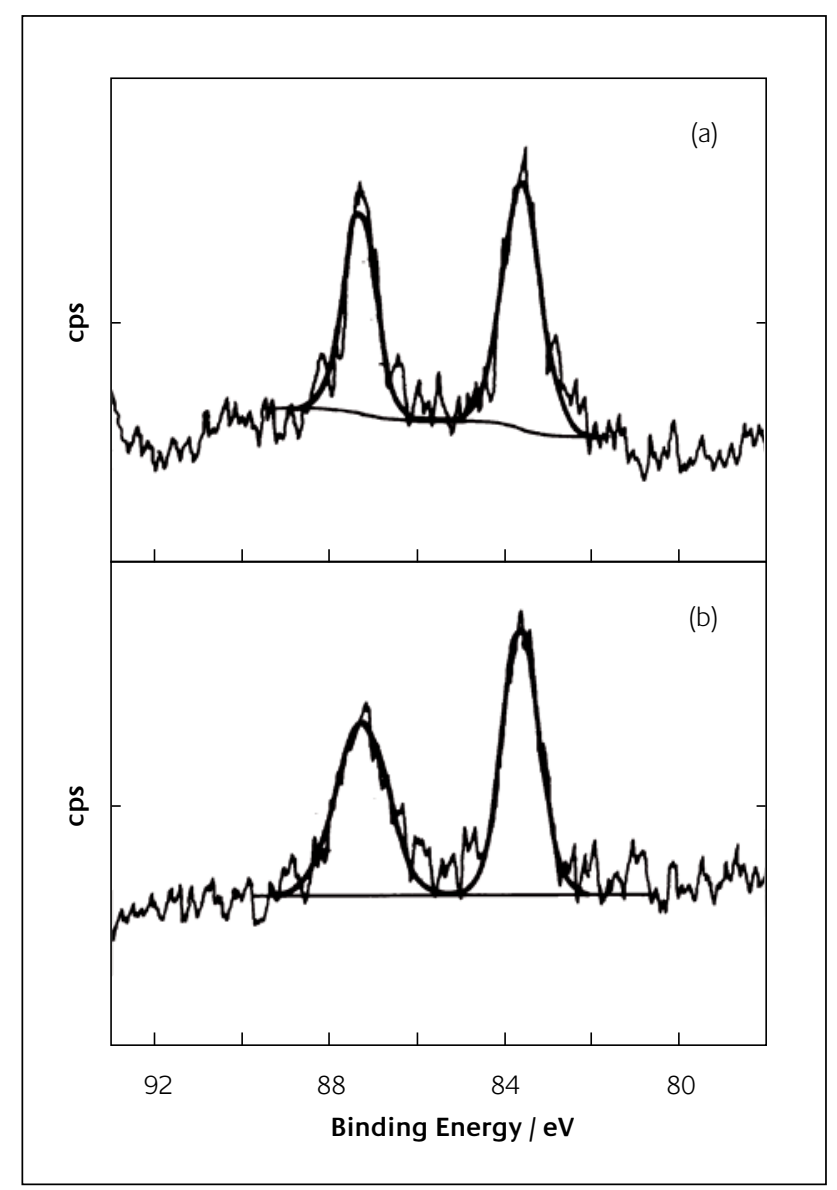

Figure 3

High resolution XPS scan of the Au (4f) region of 1 wt.\% Au/TiO, THPC (a) before irradiation and (b) after $3 \mathrm{~h}$ irradiation at $254 \mathrm{~nm}$

respectively. Similar spectra have been recently reported for THPC Au/TiO, samples, also exhibiting a shift to lower BE compared to the Au reference (26). This has been attributed to the increased fraction of surface atoms, which, being less coordinated, exhibit reduced screening of the photoelectron core hole. The small change in the peaks shape noticed after irradiation (Figure 3) might be ascribed to the presence of oxidised Au forms, as in similar $\mathrm{Au} / \mathrm{TiO}$, systems $(27,28)$. In fact, although most of the photogenerated holes are generally scavenged by surface hydroxyl groups, a fraction of them can react with metallic gold on the photocatalyst surface, converting it into $A u(I)$ and possibly then into $A u(I I I)$, the oxidation potential of the holes photogenerated in the semiconductor valence band $\left(\mathrm{E}_{\mathrm{VB}} \sim 2.5 \mathrm{~V}\right)$ being more positive than that of metallic gold $\left(\mathrm{E}^{0}\left(\mathrm{Au} / \mathrm{Au}^{+}\right)=1.76 \mathrm{~V}\right)(5)$

The effect of gold deposition was also examined by HRTEM analysis, yielding information on the dimension and the distribution of the Au nanoparticles on the $\mathrm{TiO}_{2}$ surface. As reported in Table 1, the mean diameter of gold nanoparticles in $1 \mathrm{wt} . \% \mathrm{Au} / \mathrm{TiO}_{2}$ varied between 3.5 and $4.2 \mathrm{~nm}$, depending on the preparation procedure, in agreement with previous results for similar Au loadings (29). The preparative methods employed using Au sols as starting materials guarantee the maintenance of $\mathrm{Au}$ particle size in the deposition step on the $\mathrm{TiO}_{2}$ surface, as already verified on different supports (11).

\section{Photocatalytic activity}

Preliminary tests, performed to verify if gold nanoparticles affect AR1 adsorption on the semiconductor, revealed that ca. $28 \%$ of the azo dye $\left(2.5 \times 10^{-5} \mathrm{M}\right)$ was adsorbed on the unmodified titanium dioxide surface $\left(1.0 \mathrm{~g} \mathrm{l}^{-1}\right)$ and only ca. $17 \%$ on the Au modified surface $\left(1.0 \mathrm{~g} \mathrm{l}^{-1}\right.$ of the $1 \mathrm{wt} . \%$ $\mathrm{Au} / \mathrm{TiO}_{2}$ DP sample). The iso-electric point of Au-modified $\mathrm{TiO}_{2}$ has been reported to shift to lower $\mathrm{pH}$ values (17) with respect to unmodified $\mathrm{TiO}_{2}$, thus extending the $\mathrm{pH}$ region where the photocatalyst surface is negatively charged. Of course this inhibits the adsorption of negatively charged compounds, such as the bisulfonic azo dye AR1. Moreover, Au deposition can also alter $\mathrm{TiO}_{2}$ surface speciation. Recent DRIFTS studies indicated a substantial decrease of the surface amount of $-\mathrm{OH}$ groups in the presence of nanoscopic gold particles, especially for high Au loadings (21).

In order to identify the most suitable Au deposition method for the preparation of efficient $\mathrm{Au} / \mathrm{TiO}_{2}$ photocatalysts, the activity of the various $1 \mathrm{wt} . \% \mathrm{Au} / \mathrm{TiO}_{2}$ samples, prepared using the different methods, was first compared. Prior to their use as photocatalysts, all PVA and THPC samples underwent a pre-treatment step, consisting in a $3 \mathrm{~h}$ - long pre-irradiation at $254 \mathrm{~nm}$ of their water suspensions in the absence of substrate. In this way, the gold nanoparticle stabilisers, i.e. polyvinylalcohol or tetrakis(hydroxymethyl)phosphonium chloride, could be efficiently removed photocatalytically. Indeed, both types of $\mathrm{Au} / \mathrm{TiO}_{2}$ photocatalysts exhibited a much lower activity in AR1 photocatalytic degradation if they were employed without any pre-treatment (see for example Figure 4). Longer pre-irradiation did not improve the photocatalytic activity of PVA and THPC samples. By contrast, DP samples did not require any pre-irradiation treatment, no organics having been used in their preparation. Indeed, AR1 photodegradation on pre-irradiated $\mathrm{DP} \mathrm{Au} / \mathrm{TiO}_{2}$ proceeded at exactly the same rate as on not pre-treated DP $\mathrm{Au} / \mathrm{TiO}_{2}$ (Figure 4).

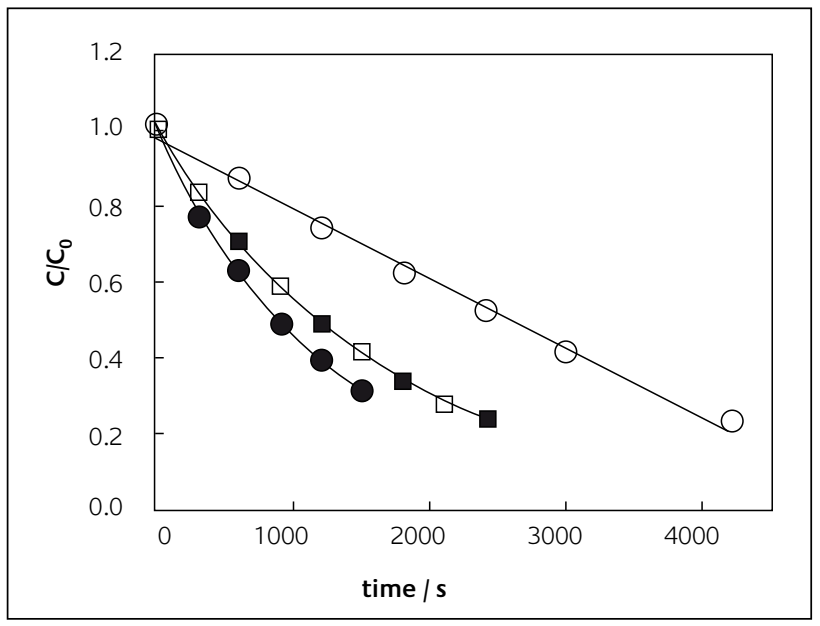

Figure 4

Effect of $3 \mathrm{~h}$ - long pre-irradiation at $254 \mathrm{~nm}$ of $\mathrm{Au} / \mathrm{TiO}_{2}$ (1 wt.\%) on AR 1 photocatalytic degradation: DP (squares) and THPC (circles) as synthesised (open symbols) and after $3 \mathrm{~h}$ - long pre-irradiation (full symbols) 
The first order rate constants of AR1 photocatalytic degradation reported in Table 1 indicate THPC Au/TiO, as the best performing photocatalyst, with the PVA and DP photocatalyst samples being progressively less efficient, in that order. Most probably, the lower activity of DP compared to THPC and PVA Au/TiO 2 is derived from the calcination step at $400^{\circ} \mathrm{C}$, required for reducing $\mathrm{Au}(\mathrm{III})$ to $\mathrm{Au}(0)$ only in the DP preparation route. Calcination not only reduces the surface area and the quantity of $\mathrm{OH}$ surface groups, but, in the case of $\mathrm{TiO}_{2}$, may also induce some conversion of the anatase phase into the rutile phase, which might be less photoactive (1). Moreover, heat treatment may also increase the gold particles size (30). Indeed, the rate constant of AR1 photodegradation on a PVA Au/ $\mathrm{TiO}_{2}$ sample, which had been calcined at $400^{\circ} \mathrm{C}$, was ca. $25 \%$ lower than that obtained employing a non calcined PVA sample.

The effect of the Au loading, deposited on the $\mathrm{TiO}_{2}$ surface through the most efficient THPC method, was investigated in the $0.5-20 \mathrm{wt} . \% \mathrm{Au} / \mathrm{TiO}_{2}$ range. The first order rate constants of AR1 photocatalytic degradation are collected in Table 2. An increase of the photocatalytic efficiency was obtained by increasing the amount of gold up to $5 \mathrm{wt} . \%$; and in this range the rate constants of AR 1 photodegradation were equal to $\left(0.5 \mathrm{wt} . \% \mathrm{Au} / \mathrm{TiO}_{2}\right.$ ) or higher $(0.7,1.0$ and $5.0 \mathrm{wt} . \% \mathrm{Au} / \mathrm{TiO}_{2}$ ) than that obtained on titanium dioxide containing no gold. Thus, low gold loadings are not very effective in reducing the rate of recombination between the conduction band electrons and the valence band holes and a substantial increase of photoactivity can be attained for Au loadings above 1 wt.\%. In contrast, very high $\mathrm{Au}$ loadings, e.g. 20 wt.\% $\mathrm{Au}$ on $\mathrm{TiO}_{2}$, had a negative effect on the photodegradation rate, mainly due to lower substrate adsorption on the oxide surface and to screening effects, with the metal deposits reducing the direct absorption of light by titanium dioxide and thus the photogeneration of charged species. Moreover, Au

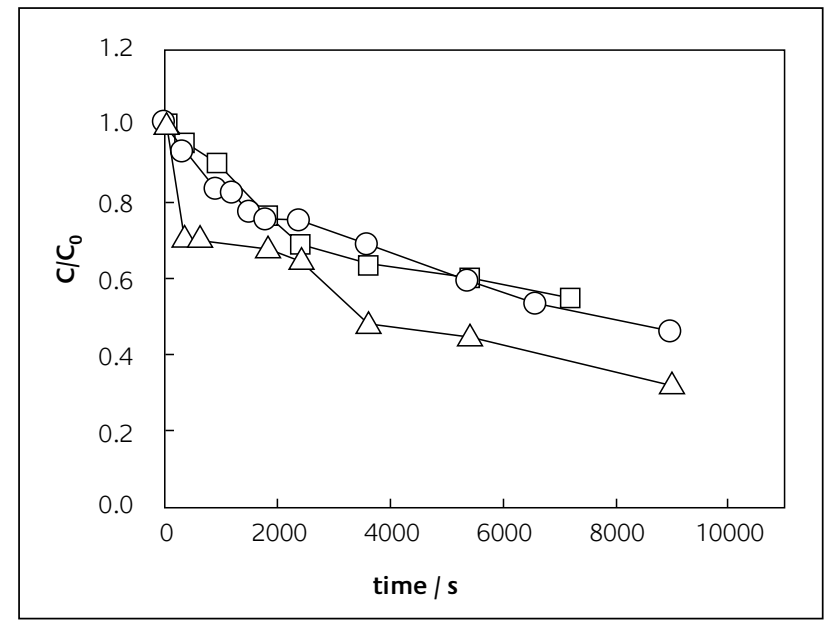

\section{Figure 5}

Effect of Au loading on the photocatalytic mineralization of AR1: unmodified $\mathrm{TiO}_{2}$ (squares), 1 wt.\% (circles) and 5 wt.\% (triangles) $\mathrm{Au} / \mathrm{TiO}_{2}$ THPC
Table 2: Effect of Au loading of Au/TiO 2 THPC photocatalysts on the first order rate constants of AR1 photocatalytic degradation

\begin{tabular}{|c|c|}
\hline Au loading / wt.\% & $10^{4} \times \mathrm{k} / \mathrm{s}^{-1}$ \\
\hline 0 & $5.8 \pm 0.5$ \\
\hline 0.5 & $5.6 \pm 0.2$ \\
\hline 0.7 & $6.2 \pm 0.5$ \\
\hline 1.0 & $7.0 \pm 0.7$ \\
\hline 5.0 & $8.6 \pm 0.6$ \\
\hline 20 & $3.7 \pm 0.3$ \\
\hline
\end{tabular}

nanoparticles can also act as effective scavengers of photogenerated holes (vide infra), inhibiting their reaction with the substrate (19).

The photocatalytic degradation of organic water pollutants, such as AR1, notoriously produces intermediate species, whose toxicity may be higher than that of the starting compound. Thus, the extent of mineralization, i.e. the effective conversion of organic compounds into $\mathrm{CO}_{2}$, $\mathrm{H}_{2} \mathrm{O}$ and mineral acids, should always be checked. Figure 5 gives the AR1 photomineralization profiles in unmodified $\mathrm{TiO}_{2}$ and in 1 and 5 wt.\% Au/TiO 2 THPC suspensions, as monitored by total organic carbon (TOC) analysis. Of course, AR1 photomineralization proceeded at a much lower rate than AR1 photodegradation; in fact, as shown in Figure 5 , a $50 \%$ reduction of the TOC content was attained after a ca. 5-fold longer irradiation time, respect to the irradiation time required to halve the AR1 initial concentration. On the other hand, the Au loading of $\mathrm{TiO}_{2}$ had the same effect in AR1 photodegradation and photomineralization, this latter proceeding on $5 \mathrm{wt} . \% \mathrm{Au} / \mathrm{TiO}_{2}$ at a higher rate than on 1 wt. $\% \mathrm{Au} / \mathrm{TiO}_{2}$, which behaved only slightly better than unmodified $\mathrm{TiO}_{2}$. Thus, the beneficial effect of $\mathrm{Au}$ nanoparticles deposition on $\mathrm{TiO}_{2}$ is not specific for the photocatalytic degradation of AR1, but it was confirmed

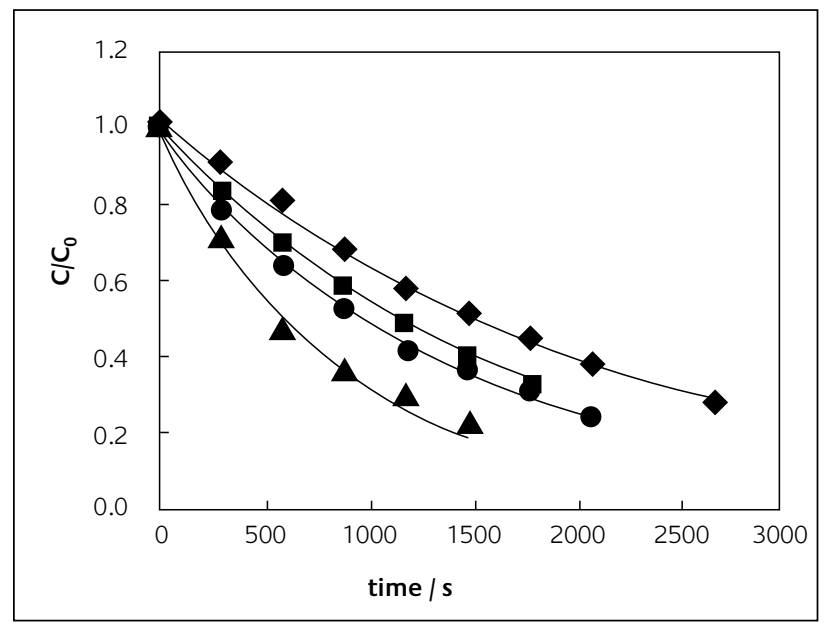

\section{Figure 6}

Effect of $\mathrm{pH}$ on AR1 photocatalytic degradation in $1 \mathrm{wt} \% \mathrm{Au} / \mathrm{TiO} \mathrm{T}_{2} \mathrm{THPC}$ suspensions: $\mathrm{pH} 3$ (diamonds), $\mathrm{pH} 4$ (squares), natural $\mathrm{pH}$ (circles) and pH 9.6 (triangles) 
also for the photocatalytic degradation of its mineralization by-products.

Finally, the effect of $\mathrm{pH}$ on the rate of AR1 photocatalytic degradation was also investigated in the presence of 1 wt.\% $\mathrm{Au} / \mathrm{TiO}_{2}$ THPC. The results obtained in the $3-10 \mathrm{pH}$ range, given in Figure 6 , should be considered in comparison with the $\mathrm{pH}$-dependence of the rate of AR1 photocatalytic degradation on unmodified $\mathrm{TiO}_{2}$, exhibiting a maximum under so-called natural $\mathrm{pH}$ conditions (31), i.e. for an initial $\mathrm{pH}$ of 5.8, corresponding to optimal conditions for electrostatic interaction between the negatively charged AR1 molecule and the positively charged $\mathrm{TiO}_{2}$ surface. Lower rates were measured under $254 \mathrm{~nm}$ irradiation either at lower or at higher $\mathrm{pH}$, the rate constant of AR1 photocatalytic degradation on $\mathrm{TiO}_{2}$ at $\mathrm{pH} 9.6$ being more than $20 \%$ lower than that measured at natural $\mathrm{pH}$, in line with the results of AR1 photocatalytic degradation on $\mathrm{TiO}_{2}$ under longer wavelength irradiation (31). Surprisingly, AR1 photodegradation on $\mathrm{Au} / \mathrm{TiO}_{2}$ proceeded significantly faster at pH 9.6 (Figure 6), i.e. the photocatalytic activity of Aumodified titanium dioxide increases with increasing the $\mathrm{OH}^{-}$ concentration in the aqueous phase.

There is indirect evidence of a reaction occurring between the surface metallic gold and the valence band holes. In fact, in the presence of a high concentration of hydroxide, hydroxyl radicals can be generated more easily by reaction with valence band holes (32). Under such conditions the hydroxide anions in the aqueous phase can compensate for the smaller concentration of hydroxyl groups on the Au modified surface (21) and act as effective scavengers for photoproduced holes. Thus, the higher photocatalytic degradation rates obtained under these conditions are a consequence of the lower probability that valence band holes oxidise $\mathrm{Au}^{0}$ to $\mathrm{Au}^{+}$, which will act as a recombination centre, in contact with conduction band electrons. The beneficial effect of gold deposition on $\mathrm{TiO}_{2}$ thus seems to be strictly related to the minimisation of the reaction between metallic Au and photogenerated holes.

\section{Conclusions}

The deposition of nanosized gold particles on the $\mathrm{TiO}_{2}$ surface may increase the photocatalytic activity of the semiconductor oxide, by increasing the efficiency of charge separation of the light-generated electron-hole pairs. The best photocatalytic activity was attained with THPC Au/TiO , after a pre-irradiation step to remove the colloidal stabiliser. Optimal Au loadings should be employed ( 5 wt.\% Au on $\mathrm{TiO}_{2}$ in the present case), possibly depending on the adsorption properties of the substrate, for ensuring optimal charge separation without excessive light screening effects of the $\mathrm{TiO}_{2}$ particles.

\section{About the authors}

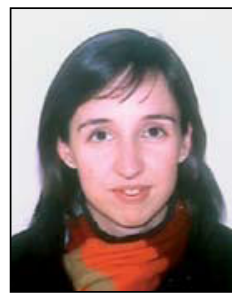

Marta Mrowetz graduated in Chemistry (2002) and completed her PhD in Chemical Sciences (2006) at the University of Milan under the supervision of Prof. E. Selli. In 2004 she spent several months at Caltech, working with A.J. Colussi and M.R. Hoffmann. Then she was awarded a post-doc position at the University of Milan. She has always been involved in the mechanistic aspects of photocatalysis on semiconductors.

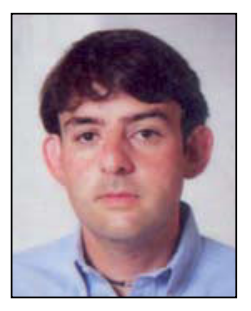

Alberto Villa presently works as a PhD student in the laboratory of Prof. Laura Prati in the Inorganic Chemistry Department at the University of Milan. $\mathrm{He}$ is a graduate in Industrial Chemistry (2004), his main interest being in catalytic oxidation reactions. The particular experience he has acquired is in the field of preparation of gold on carbon and its use in liquid phase oxidation.

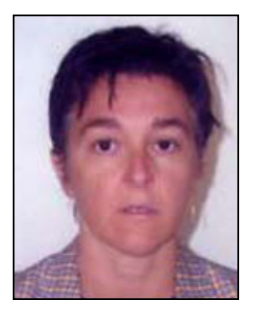

Laura Prati is presently an Associate Professor in Inorganic Chemistry at the University of Milan. She is a graduate in Chemistry (1983), received her specialisation in "Tecniche Analitiche per la Chimica Organica Fine" from Politecnico of Milan in 1985 and was awarded a PhD in Industrial Chemistry in 1988. Her main interest is in catalytic hydrogenation and oxidation reactions and she has been involved in catalytic applications for gold since 1986. Particular experience she had acquired is in the field of preparation of gold on carbon and its use in the liquid phase oxidation.

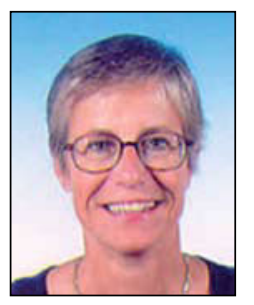

Elena Selli graduated in Chemistry at the Scuola Normale Superiore in Pisa (Italy) in 1977. She has been a PhD student and research fellow of the Scuola Normale Superiore (1981) and of the University of Milan (1983). She is now an associate professor of physical chemistry at the University of Milan. She has always been involved in mechanistic studies of photoreactions. In recent years, her interests were mainly focused on electron transfer processes occurring in photocatalytic processes on semiconductors, for both water purification and energy storage.

\section{References}

1 M. R. Hoffmann, S.T. Martin, W. Choi and D.W. Bahnemann, Chem. Rev., 1995, 95, 69

2 A. L. Linsebigler, G. Lu and J.T. Yates, Jr., Chem. Rev., 1995, 95, 735

3 F. B. Li and X.Z. Li, Chemosphere, 2002, 48, 1103

4 Z. Kasarevic-Popovic, D. Behar and J. Rabani, J. Phys. Chem. B, 2004, 108, 20291 
5 V. Subramanian, E. E. Wolf and P.V. Kamat, J. Phys. Chem. B, 2001, 105, 11439; Langmuir, 2003, 19, 469

6 A. Wood, M. Ciersig and P. Mulvaney, J. Phys. Chem. B, 2001, 105, 8810

7 M. Haruta, Catal. Today, 1997, 36, 153

8 J.-D. Grunwaldt, C. Kiener, C. Wögerbauer and A. Baiker, J. Catal., 1999, 181, 223

9 L. Prati and G. Martra, Gold Bull., 1999, 32, 96

10 D. G. Duff, A. Baiker and P. Edwards, J. Chem. Soc., Chem. Commun., 1993, 96

11 F. Porta, L. Prati, M. Rossi, S. Coluccia and G. Martra, Catal. Today, 2000, 61, 165

12 M. Bertelli and E. Selli, Appl. Catal. B: Environ., 2004, 52, 205

13 C. G. Hatchard and C.A. Parker, Proc. R. Soc. London, 1956, A235, 518

14 J. M. Joseph, H. Destaillats, H.M. Hung and M.R. Hoffmann, J. Phys. Chem. A, 2000, 104, 301

15 M. Abdullah, J.K.C. Low and R.W. Matthews, J. Phys. Chem., 1990, 94, 6820

16 M. Mrowetz, C. Pirola and E. Selli, Ultrason. Sonochem., 2003, 10, 247

17 X. Z. Li and F.B. Li, Environ. Sci. Technol., 2001, 35, 2381

18 I. M. Arabatzis, T. Stergiopoulos, D. Andreeva, S. Kitova, S.G. Neophytides and P. Falaras, J. Catal., 2003, 220, 127
19 A. Dobosz and A. Sobczynski, Monatsh. Chem., 2001, 132, 1037

20 S. Sakthivel, M. V. Shankar, M. Palanichamy, B. Arabindoo, D.W. Bahnemann and V. Murugesan, Water Res., 2004, 38, 3001

21 A. Orlov, D.A. Jefferson, N. Macleod and R. M. Lambert, Catal. Letters, 2004, 92, 41

22 U. Kreibig and M. Vollmer, Optical Properties of Metal Clusters; Springer-Verlag: Berlin, 1995

23 M. M. Alvarez, J. T. Khoury, T. G. Schaaff, M. N. Shafigullin, I. Vezmar and R. L. Whetten, J. Phys. Chem. B, 1997, 101, 3706

24 P. V. Kamat, J. Phys. Chem. B, 2002, 106, 7729

25 A. Watanabe and H. Kozuka, J. Phys. Chem. B, 2003, 107, 12713

26 X. Z. Li, C. He, N. Graham and Y. Xiong, J. Appl. Electrochem., 2005, 35,741

27 A. Zwijnenburg, A. Goossens, W. G. Sloof, M. W. J. Crajé, A. M. van der Kraan, L. Jos de Jongh, M. Makkee and J.A. Moulijn, J. Phys. Chem. B, 2002, 106, 9853

28 M. P. Casaletto, A. Longo, A. Martorana, A. Prestianni and A. M. Venezia, Surf. Interface Anal., 2006, 38, 215

29 T. Hayashi, K. Tanaka and M. Haruta, J. Catal., 1998, 178, 566

30 A. G. Shastri, A. K. Datye and J. Schwank, J. Catal., 1984, 87, 265

31 M. Mrowetz and E. Selli, J. Photochem. Photobiol. A: Chem., 2006, 180,15

32 I. K. Konstantinou and T. A. Albanis, Appl. Catal. B: Environ., 2004, 49, 1 